\title{
Sliding mode stabilisation of networked systems with consecutive data packet dropouts using only accessible information
}

\author{
Ahmadreza Argha, Li Li, and Steven W. Su \\ Email: steven.su@uts.edu.au \\ Faculty of Engineering and Information Technology \\ University of Technology Sydney \\ PO Box 123, Broadway, NSW 2007, Australia
}

9 Nov. 2016

\begin{abstract}
This paper develops a novel stabilising sliding mode (SM) for systems involving uncertainties as well as measurement data packet dropouts. In contrast to the existing literature that designs the switching function by using unavailable system states, a novel linear sliding function is constructed by employing only the available communicated system states for the systems involving measurement packet losses. This also equips us with the possibility to build a novel switching component for discrete-time sliding mode control (DSMC) by using only available system states. Finally, using a numerical example, we evaluate the performance of the designed DSMC for networked systems.
\end{abstract}

\section{Introduction}

Progressively, practising control engineers exploit real-time communication networks in the construction of control systems, establishing a new thread of control systems known as networked control systems (NCSs) [31. NCSs offer several substantial advantages, in comparison with traditional control 
systems, including lower overall cost, high reliability, smaller wiring system, as well as simple installation and maintenance etc. Communication networks, however, may induce possible random time-delays and/or data packet dropouts arising from their limited bandwidth [29], which may lead to performance degradation of the control system. As a result, to be more realistic, the random communication time-delays and data packet losses should be taken into account for the stability analysis and control synthesis of NCSs [31, 29, 37, 21, 4, 9, 5, 7]. For example, [6] considers the joint design of dynamic output feedback controller and network access assignment sequences for NCSs while sufficient communication channels are not available. Moreover, the stability analysis and controller synthesis for LTI systems exploiting a network of sensors and actuators which are triggered in groups by random events are addressed by [8]. Additionally, 35] and [34] give a unified model for dealing with several phenomena in NCSs such as the nonuniform sampling, the measurement size reduction, the transmission rate reduction, the signal quantization, and the measurement missing. A unified framework is presented in [36] for cyber-physical systems (CPSs) to cope with the randomly occurring sensor saturation, signal quantization, packet dropouts along with the medium access constraint.

Moreover, because time-delays and packet losses in communication networks are mainly time-varying by nature, the existing techniques for the design of robust control systems dealing with deterministic time-delays may not be beneficial [28]. Several mathematical schemes have been employed to model the random network time-delays and packet losses with regard to the characteristics of sources and destinations. The so-called Bernoulli distribution has widely been used to model network packet losses, due to its practicality and simplicity [27, 13, 23.

Sliding Mode Control (SMC) is a well-known control strategy which can be considered as a special thread of Variable Structure Control (VSC). The basic idea of SMC is to steer the system state trajectories onto a pre-designed sliding manifold and remain there thereafter. For the design of sliding surface, a wide range of approaches has been developed, such as eigenstructure assignment, pole placement, optimal quadratic methods [3]. Moreover, LMI approaches have been proposed in [2, 10, 30]. However, traditionally SMC has been designed for the systems not involving random time-delays and packet losses; see e.g. [33, 14, 2]. Recently, several research papers have studied the problem of SMC design for networked systems with packet losses. For instance, 32 develops an integral SMC in continuous-time for offshore 
steel jacket platforms involving state time-delays. Moreover, designing the discrete-time SMC (DSMC) for NCSs with packet losses is considered in [18]. Nonetheless, the existing DSMC in the literature suffers from the following major drawbacks:

- The switching function is designed based on the system states which, due to the occurrence of packet loss in the communication channel, may not be accessible; cf. [18], [1].

- The existing approaches employ several inequalities to provide a sufficient LMI condition derived for stability analysis of the obtained closed-loop system and design the switching surface, which may lead to a very conservative LMI condition.

The main objective of this paper is to stabilise an uncertain NCS with consecutive data packet losses using sliding mode control approach while resolving the aforementioned drawbacks. In doing so, a novel sliding function is introduced by employing the available communicated system states involving packet losses, which is substantially different from the existing DSMC in the literature; see [18, 1]. In addition to this substantial contribution, this paper includes the following innovations:

- The proposed DSMC is applicable to unstable systems directly and obviates the need to pre-stabilisation step; cf. [18, 19].

- A novel LMI-based stability condition is developed for sliding surface design which has a wider region of applicability relative to the existing approach in the literature.

- Assuming an underlying system involving smooth and bounded exogenous disturbances, a novel stochastic disturbance observer is developed. A more practical switching function is then provided in the controller using the proposed disturbance estimator with the aid of the sign function. The proposed DSMC that employs the novel switching function has effectively better performance in terms of reducing the boundary layer thickness in comparison to the linear controller.

The rest of this paper is organised as follows: The problem formulation and preliminaries are given in Section 2. Section 3 is devoted to the problem of stochastic sliding surface design. A novel variable structure discontinuous component (VSDC) for DSMC is introduces in Section 4. In Section 5, the effectiveness of the given theory is evaluated through numerical examples. Finally, Section 6 will conclude this paper.

Notation We denote the symmetric elements of a symmetric block matrix by $\{\star\} . r(\cdot)$ denotes the rank of a matrix. $\|\cdot\|$ is the Euclidean norm. 
$\mathbb{E}\{\cdot\}$ denotes the mathematical expectation of a stochastic variable. Prob $\{\cdot\}$ stands for the occurrence probability of an event. $\lambda_{\min }(\cdot)\left(\lambda_{\max }(\cdot)\right)$ is used for denoting the smallest (largest) eigenvalue of a matrix.

\section{Problem Formulation and Preliminaries}

Let us consider the following uncertain linear system,

$$
x(k+1)=[G+\Delta G(k)] x(k)+H[u(k)+d(k)],
$$

where the vectors $x(k) \in \mathbb{R}^{n}, u(k) \in \mathbb{R}^{m}$ and $d(k) \in \mathbb{R}^{m}$ are the system states, control inputs, and external disturbances, respectively. We also assume that $m \leq n$, the control input matrix $H$ is full rank, and further, the matrix pair $(\boldsymbol{G}, \boldsymbol{H})$ is stabilisable. The mismatched uncertainty matrix $\Delta G(k)$ is of the form $\Delta G(k)=\mathscr{E} \mathscr{F}(k) \mathscr{G}$, in which $\mathscr{E}$ and $\mathscr{G}$ are known matrices and besides $\mathscr{F}(k)$ is an unknown matrix satisfying $\|\mathscr{F}(k)\| \leq 1, \forall k \geq 0$. The external disturbance is assumed to be bounded by a known bound, i.e. $\|d(k)\| \leq \bar{d}$.

In the developed control system, the measured signals are transmitted to the controller utilising a communication network subject to data packet losses. We exploit the following estimator to provide the communicated system states, dented by $x_{c}(\cdot) \in \mathbb{R}^{n}$ in order to distinguish it from the system states $x(\cdot)$, for the controller:

$$
x_{c}(k)=(1-\beta(k)) x(k)+\beta(k) x_{c}(k-1),
$$

where the stochastic scalar variable $\beta(k)$ is Bernoulli distributed white sequence with

$$
\begin{aligned}
& \operatorname{Prob}\{\beta(k)=1\}=\mathbb{E}\{\beta(k)\}=\bar{\beta} \\
& \operatorname{Prob}\{\beta(k)=0\}=1-\mathbb{E}\{\beta(k)\}=1-\bar{\beta},
\end{aligned}
$$

where $0 \leq \bar{\beta}<1$ implies the data packet dropout probability in the communication channel.

Remark 1. The estimation scheme (2), employed in e.g. [1], is essentially different from the one which has frequently been used in the literature [23, 27, 29, 17, 18]. Indeed, when a packet dropout occurs, this scheme provides the last available system state estimate $\left(x_{c}(k-1)\right)$, rather than the last system state $x(k-1)$ which may not necessarily be available [23, 27, 29, 17, 18], for 
the controller. Instead, the aforementioned references employ a measurement model as

$$
x_{c}(k)=(1-\beta(k)) x(k)+\beta(k) x(k-1) .
$$

The above estimation scheme is valid only if packet dropouts in the communication channel do not happen consecutively, which does not seem to be a very practical assumption. The scheme (2) can alternatively deal with the successive packet losses. Employing the model (2), when a packet loss occurs, the controller receives the last available system information, i.e. $x_{c}(k)=x_{c}(k-1)$, otherwise, the controller utilises the new arrival system information, i.e. $x_{c}(k)=x(k)$. Notice also that $x_{c}(k-1)$ in the model $(2)$ can be $x(k-1)$, or $x(k-2)$ etc. While in this estimation scheme the number of possible consecutive packet losses is not bounded, an implicit stochastic constraint would bound the number of consecutive losses, indicated by the Bernoulli variable $\beta(k)$ as $\operatorname{Prob}\{\beta(k)=1\}=\mathbb{E}\{\beta(k)\}=\bar{\beta}$; i.e. if $\bar{\beta}$ is a small value, the number of possible consecutive dropouts decreases and vice versa. The scheme (2) has also the ability to cope with the communication random delays in the discrete-time systems. If the sampling period is long compared with $\tau_{d}$ (communication random time-delay), the controller would receive the system state's information at $k$-th sampling time, i.e. $x_{c}(k)=x(k)$. However, if $\tau_{d}$ is longer than one sampling period, then $x_{c}(k)=x_{c}(k-1)$. The controller, in such a case, utilises the last available information. Here $x_{c}(k-1)$ denotes literally $x\left(k-\tau_{d}\right)$.

In what follows, the brief $\beta$ and $\Delta G$ are used rather than $\beta(k)$ and $\Delta G(k)$, respectively. Moreover, the remaining of this section is devoted to recalling some materials from the literature which will be employed in what follows in this paper.

Note that a matrix inequality problem involving an uncertain negative quadratic term is not easy to be directly converted to an LMI problem. To deal with this issue, using auxiliary inequalities seems to be beneficial, but it could also impose a great loss on the problem. This note alternatively uses a lossless method to convert the matrix inequality to a form that can be simply arranged as an LMI. Indeed, this technique extremely widens the applicability region of the given DSMC compared with the existing methods for the DSMC [18]. 
Lemma 2.1 ([15]). Consider the following inequality:

$$
\Pi(Y)-\Omega^{T}(Y) \Theta^{-1}(Y) \Omega(Y)<0 .
$$

The feasibility of the above inequality, with respect to the variable $Y$, is equivalent to the feasibility of

$$
\Pi(Y)+\mathrm{F}^{T} \Theta(Y) \mathrm{F}+\mathrm{F}^{T} \Omega(Y)+\Omega^{T}(Y) \mathrm{F}<0,
$$

with respect to the variables $Y$ and $\mathrm{F}$, in which $\Pi(Y), \Theta(Y)$ and $\Omega(Y)$ are functions of $Y, \Theta(Y)>0$, and further $\mathrm{F}$ is an auxiliary matrix.

Lemma 2.2 ([11]). Consider a given matrix $W \in \mathbb{R}^{p \times q}$ with $r(W)=q$, and

$$
W=U_{w}\left[\begin{array}{c}
\Sigma_{w} \\
0
\end{array}\right] V_{w}^{T},
$$

where $V_{w} \in \mathbb{R}^{q \times q}$ and $\boldsymbol{U}_{w} \in \mathbb{R}^{p \times p}$ are orthogonal matrices, and $\Sigma_{w}:=\operatorname{diag}\left(\sigma_{w, 1}, \cdots, \sigma_{w, q}\right)$, with $\sigma_{w, i},(i=1, \cdots, q)$ denoting nonzero singular values of $W$. Now, supposing that $0<X \in \mathbb{R}^{p \times p}$, it can be shown that there exists an invertible matrix $\mathscr{Z} \in \mathbb{R}^{q \times q}$ such that

$$
X W=W \mathscr{Z},
$$

if and only if $X$ is of the following structure

$$
X=U_{w}\left[\begin{array}{cc}
X_{11} & 0 \\
0 & X_{22}
\end{array}\right] U_{w}^{T},
$$

where $0<X_{11} \in \mathbb{R}^{q \times q}$ and $0<X_{22} \in \mathbb{R}^{(p-q) \times(p-q)}$.

\section{$3 \quad$ Sliding Surface Design}

Consider the following stochastic discrete-time switching surface,

$$
\mathcal{S}=\left\{x_{c}: s_{c}(k) \triangleq F x_{c}(k)=0\right\},
$$

where the matrix $F \in \mathbb{R}^{m \times n}$ will be derived later in this paper so that it ensures the non-singularity of $\boldsymbol{F} \boldsymbol{H}$. It is worth mentioning that the switching surface introduced in $(9)$ is essentially different from the one proposed in 
[18, 1] for NCSs involving packet losses. The proposed sliding surface in [18] ([1]) is constructed using the system states $x(k)$ and $x(k-2)\left(x_{s}(k-2)\right)$, and cannot be employed directly in the VSDC strategy, since because of the packet loss in the communication network, $x(k)$ and $x(k-2)$ are not accessible.

Let $d(k)=\left[d_{1}(k), \cdots, d_{m}(k)\right]^{T}$, and $\bar{d}_{i} \leq d_{i}(k) \leq \underline{d}_{i}, \quad i=1, \cdots, m$. We define

$$
d_{i, a}=\frac{\bar{d}_{i}+\underline{d}_{i}}{2}, d_{i, b}=\frac{\bar{d}_{i}-\underline{d}_{i}}{2}, \quad i=1, \cdots, m,
$$

and introduce

$$
\mathscr{D}_{a}=\left[d_{1, a}, \cdots, d_{m, a}\right]^{T}, \mathscr{D}_{b}=\left[d_{1, b}, \cdots, d_{m, b}\right]^{T} .
$$

Now, consider the following control law:

$$
u(k)=-(F H)^{-1} F G x_{c}(k)-\psi(k),
$$

where $\psi(k)$ is a component to minimise the harmful influence of the exogenous disturbance $d(k)$ on the boundary layer thickness. Denoting $d_{\psi}(k) \triangleq$ $d(k)-\psi(k)$, the component $\psi(k)$ is assumed to be bounded while satisfying

$$
\left\|d_{\psi}(k)\right\| \leq \varpi\left\|\mathscr{D}_{b}\right\|,
$$

where $\varpi$ is a positive scalar. It is worth mentioning that the component $\psi$ has the following general form in the literature:

$$
\psi(k)=\varsigma+\rho \operatorname{sgn}\left(s_{c}(k)\right),
$$

where $\varsigma$ and $\rho$ are two constants associated with the bounds of $\boldsymbol{F} \boldsymbol{H d}(k)$, i.e. $\mathscr{D}_{a}$ and $\operatorname{diag}\left(\mathscr{D}_{b}\right)$ where $\operatorname{diag}\left(\mathscr{D}_{b}\right)=\operatorname{diag}\left(d_{1, b}, \cdots, d_{m, b}\right)$ (see (11)). Nevertheless, it should be mentioned that exploiting the above discontinuous variable structure component in a discrete-time control law leads the system state trajectories to chatter around the sliding surface with a frequency equal to the digital system's sampling frequency and thus a boundary layer with thickness $O(T)$ [25]. On the other hand, with the assumption of boundedness and smoothness of the exogenous disturbance, a different manner has been developed in the literature for deterministic DSMC, using the idea of disturbance estimation. A novel choice for $\psi(k)$ will be presented later in Section 4 employing a disturbance observer scheme which is specifically suitable for 
stochastic systems. We apply the control law in (12) to the system (1) to obtain the closed-loop system as

$$
x(k+1)=[G+\Delta G-(1-\beta) \hat{G}] x(k)-\beta \hat{G} x_{c}(k-1)+H d_{\psi}(k),
$$

where $\hat{\boldsymbol{G}}=\boldsymbol{H}(\boldsymbol{F H})^{-1} \boldsymbol{F} \boldsymbol{G}$. In addition, it can be seen that

$$
\begin{aligned}
s_{c}(k+1)= & \left(1-\beta_{k+1}\right)\left[\beta_{k} F(G+\Delta G)+\left(1-\beta_{k}\right) F \Delta G\right] x(k)-\beta_{k}\left(1-\beta_{k+1}\right) F G x_{c}(k-1) \\
& +\left(1-\beta_{k+1}\right) F H d_{\psi}(k)+\beta_{k+1}\left(1-\beta_{k}\right) F x(k)+\beta_{k+1} \beta_{k} F x_{c}(k-1) .
\end{aligned}
$$

\subsection{Stability analysis}

This subsection analyses the stability of the closed-loop system (15) as well as the derived sliding motion dynamics (16). It is worth noting that in terms of dealing with systems involving exogenous disturbances, the DSMC can only guarantee the system state trajectories ultimately enter into a boundary layer about $\mathcal{S}=\left\{x_{c}: F x_{c}(k)=0\right\}$. Moreover, as the system (1) involves the mismatched uncertainty $\Delta G$, reachability analysis of the quasi-sliding mode (QSM) using a separate sufficient condition may not be possible. As an alternative scheme to analyse simultaneously the reachability of the QSM in addition to the stability of the system states, whilst the exogenous disturbance is absent, the following theorem is given. The boundedness characterisation of the augmented system states will be carried out later in Theorem 3.2 (see Subsection 3.2. Nonetheless, since we also need to consider the cross-terms between the overall system state and $d_{\psi}(k)$ in Theorem 3.2 , the proof of Theorem 3.1 is done by including the external disturbance and the term $d_{\psi}(k)$. This can help us avoid repetition of the technical manipulations in Theorem 3.2. Eventually, letting $\left[\begin{array}{l}d_{\psi}(k) \\ \psi(k)\end{array}\right]=0$, the sufficient LMI stability condition exploited for the controller synthesis purposes will be obtained. Notice also that the LMI-based stability condition proposed in the following theorem to design the sliding manifold is constructed based on the notion of exponential mean-square stability; see e.g. Definition 1 in [29].

Theorem 3.1. When no external disturbances exist in the system (1), the control law (12) drives the state trajectories onto the switching surface introduced in (9), and the closed-loop system state is exponentially mean-square 
stable, if there exist $P:=U\left[\begin{array}{cc}P_{11} & 0 \\ 0 & P_{22}\end{array}\right] U^{T}>0, Q>0, X_{i}, i=1,2,3$, and scalar variables $\epsilon>0, v>0$ satisfying the following $L M I$ :

$$
\left[\begin{array}{cccccccc}
\check{\Psi}_{11} & \star & \star & \star & \star & \star & \star & \star \\
0 & \check{\Psi}_{22} & \star & \star & \star & \star & \star & \star \\
\mu_{1} H^{T} P G & -\mu_{1} H^{T} P G & -H^{T} P H & \star & \star & \star & \star & \star \\
0 & 0 & 0 & -H^{T} P H & \star & \star & \star & \star \\
H X_{2} & 0 & 0 & 0 & -P & \star & \star & \star \\
P G+H X_{1} & 0 & 0 & 0 & 0 & -P & \star & \star \\
0 & H X_{3} & 0 & 0 & 0 & 0 & -P & \star \\
0 & 0 & \mu_{1} \mathscr{E}^{T} P H & \mu_{2} \mathscr{E}^{T} P H & 0 & \mathscr{E}^{T} P & 0 & -\epsilon I
\end{array}\right]<0,
$$

where $\check{\Psi}_{11}=-P+\bar{Q}+(1-\bar{\beta})\left(X_{2}^{T} H^{T}+H X_{2}\right)+v I+\epsilon \mathscr{G}^{T} \mathscr{G}, \quad \check{\Psi}_{22}=-\bar{Q}+$ $\delta\left(X_{3}^{T} H^{T}+H X_{3}\right)+v I, \mu_{1}=\sqrt{\bar{\beta}(2-\bar{\beta})}, \quad \mu_{2}=\sqrt{(1-\bar{\beta})(2-\bar{\beta})}, \delta=\sqrt{\bar{\beta}(1-\bar{\beta})}$ and $\bar{Q}=(1-\bar{\beta}) Q$. Also, $U$ is an orthogonal matrix coming from singular value decomposition of $\boldsymbol{H}$ (refer to (7) in Lemma 2.2), and $\boldsymbol{F}=\boldsymbol{H}^{T} \boldsymbol{P}$.

Proof. Define the Lyapunov candidate function as

$$
V(\xi(k))=x^{T}(k) P x(k)+x_{c}^{T}(k-1) Q x_{c}(k-1)+s_{c}^{T}(k)(F H)^{-1} s_{c}(k),
$$

where the augmented system state vector is $\xi(k)=\left[\begin{array}{lll}x^{T}(k) & x_{c}^{T}(k-1) & s_{c}^{T}(k)\end{array}\right]^{T}$, $P>0$ and $Q>0$ are symmetric matrices and sliding function matrix $F$ is considered to be $\boldsymbol{F}=\boldsymbol{H}^{T} \boldsymbol{P}$. Notice that as $\boldsymbol{P}>0, \boldsymbol{F} \boldsymbol{H}=\boldsymbol{H}^{T} \boldsymbol{P} \boldsymbol{H}$ is invertible. Then, defining $\Delta V(\zeta(k)) \triangleq \mathbb{E}\{V(\xi(k+1)) \mid \xi(k)\}-V(\xi(k))$, it can be shown that

$$
\begin{aligned}
\Delta V(\xi(k))= & \mathbb{E}\left\{x^{T}(k+1) P x(k+1)+x_{c}^{T}(k) Q x_{c}(k)+s_{c}^{T}(k+1)(F H)^{-1} s_{c}(k+1) \mid \xi(k)\right\} \\
& -x^{T}(k) P x(k)-x_{c}^{T}(k-1) Q x_{c}(k-1)-s_{c}^{T}(k)(F H)^{-1} s_{c}(k) .
\end{aligned}
$$

It follows then

$$
\begin{aligned}
& \mathbb{E}\left\{x^{T}(k+1) P x(k+1) \mid \xi(k)\right\} \\
= & \mathbb{E}\left\{x^{T}(k)[G+\Delta G-(1-\beta) \hat{G}]^{T} P[G+\Delta G-(1-\beta) \hat{G}] x(k)\right. \\
& -2 x^{T}(k)[G+\Delta G-(1-\beta) \hat{G}]^{T} P \beta \hat{G} x_{c}(k-1) \\
& +\beta^{2} x_{c}^{T}(k-1) G^{T} F^{T}(F H)^{-1} F G x_{c}(k-1) \\
& +2 x^{T}(k)[G+\Delta G-(1-\beta) \hat{G}]^{T} F^{T} d_{\psi}(k) \\
& \left.-2 \beta x_{c}^{T}(k-1) G^{T} F^{T} d_{\psi}(k)+d_{\psi}^{T}(k)(F H) d_{\psi}(k) \mid \xi(k)\right\} .
\end{aligned}
$$


Additionally as $\mathbb{E}\{\beta(1-\beta)\}=0, \mathbb{E}\left\{\beta^{2}\right\}=\bar{\beta}$ and $\mathbb{E}\left\{(1-\beta)^{2}\right\}=1-\bar{\beta}$, we have

$$
\begin{aligned}
& \mathbb{E}\left\{x^{T}(k)\left[G+\Delta G-(1-\beta) \hat{G}_{\Delta}+(1-\beta) \Delta \hat{G}\right]^{T} P\right. \\
& \left.\times\left[G+\Delta G-(1-\beta) \hat{G}_{\Delta}+(1-\beta) \Delta \hat{G}\right] x(k) \mid \xi(k)\right\} \\
= & x^{T}(k)\left[(G+\Delta G)^{T} P(G+\Delta G)-(1-\bar{\beta})(G+\Delta G)^{T} F^{T}(F H)^{-1} F(G+\Delta G)\right. \\
& \left.+(1-\bar{\beta}) \Delta G^{T} F^{T}(F H)^{-1} F \Delta G\right] x(k),
\end{aligned}
$$

where $\hat{G}_{\Delta}=H(F H)^{-1} F(G+\Delta G), \Delta \hat{G}=H(F H)^{-1} F \Delta G$, and

$$
\begin{gathered}
\mathbb{E}\left\{-2 x^{T}(k)[G+\Delta G-(1-\beta) \hat{G}]^{T} P \beta \hat{G} x_{c}(k-1) \mid \xi(k)\right\} \\
=-2 \bar{\beta} x^{T}(k)(G+\Delta G)^{T} F^{T}(F H)^{-1} F G x_{c}(k-1), \\
\mathbb{E}\left\{\beta^{2} x_{c}^{T}(k-1) G^{T} F^{T}(F H)^{-1} F G x_{c}(k-1) \mid \xi(k)\right\} \\
=\bar{\beta} x_{c}^{T}(k-1) G^{T} F^{T}(F H)^{-1} F G x_{c}(k-1), \\
\mathbb{E}\left\{2 x^{T}(k)\left[G+\Delta G-(1-\beta) \hat{G}_{\Delta}+(1-\beta) \Delta \hat{G}\right]^{T} F^{T} d_{\psi}(k) \mid \xi(k)\right\} \\
=2 x^{T}(k)(\bar{\beta} G+\Delta G)^{T} F^{T} d_{\psi}(k), \\
\mathbb{E}\left\{-2 \beta x_{c}^{T}(k-1) G^{T} F^{T} d_{\psi}(k) \mid \xi(k)\right\} \\
=-2 \bar{\beta} x_{c}^{T}(k-1) G^{T} F^{T} d_{\psi}(k) .
\end{gathered}
$$

In addition, it can readily be demonstrated that

$$
\begin{aligned}
& \mathbb{E}\left\{x_{c}^{T}(k) Q x_{c}(k) \mid \xi(k)\right\} \\
= & (1-\bar{\beta}) x^{T}(k) Q x(k)+\bar{\beta} x_{c}^{T}(k-1) Q x_{c}(k-1),
\end{aligned}
$$

$$
\begin{aligned}
& \mathbb{E}\left\{s_{c}^{T}(k+1)(F H)^{-1} s_{c}(k+1) \mid \xi(k)\right\} \\
= & \delta^{2} x^{T}(k)(G+\Delta G)^{T} F^{T}(F H)^{-1} F(G+\Delta G) x(k) \\
& +(1-\bar{\beta})^{2} x^{T}(k) \Delta G^{T} F^{T}(F H)^{-1} F \Delta G x(k) \\
& -2 \delta^{2} x^{T}(k)(G+\Delta G)^{T} F^{T}(F H)^{-1} F G x_{c}(k-1)+2 \delta^{2} x^{T}(k)(G+\Delta G)^{T} F^{T} d_{\psi}(k) \\
& +2(1-\bar{\beta})^{2} x^{T}(k) \Delta G^{T} F^{T} d_{\psi}(k)+\delta^{2} x_{c}^{T}(k-1) G^{T} F^{T}(F H)^{-1} F G x_{c}(k-1) \\
& -2 \delta^{2} x_{c}^{T}(k-1) G^{T} F^{T} d_{\psi}(k)+(1-\bar{\beta}) d_{\psi}^{T}(k)(F H) d_{\psi}(k)+\delta^{2} x^{T}(k) F^{T}(F H)^{-1} F x(k) \\
& +\bar{\beta}^{2} x_{c}^{T}(k-1) F^{T}(F H)^{-1} F x_{c}(k-1),
\end{aligned}
$$




$$
\begin{aligned}
& \mathbb{E}\left\{s_{c}^{T}(k)(F H)^{-1} s_{c}(k) \mid \xi(k)\right\} \\
= & (1-\bar{\beta}) x^{T}(k) F^{T}(F H)^{-1} F x(k)+\bar{\beta} x_{c}^{T}(k-1) F^{T}(F H)^{-1} F x_{c}(k-1),
\end{aligned}
$$

in which $\mathbb{E}\left\{\beta_{k+1} \beta_{k}\right\}=\bar{\beta}^{2}, \mathbb{E}\left\{\left(1-\beta_{k+1}\right) \beta_{k}\right\}=\mathbb{E}\left\{\left(1-\beta_{k}\right) \beta_{k+1}\right\}=\bar{\beta}(1-\bar{\beta}) \triangleq \delta^{2}$ and $\mathbb{E}\left\{\left(1-\beta_{k}\right)\left(1-\beta_{k+1}\right)\right\}=(1-\bar{\beta})^{2}$. Employing the inequalities (19)-(27), it follows from $(18)$ that

$$
\Delta V(\xi(k))=\left[\begin{array}{c}
x(k) \\
x_{c}(k-1) \\
d_{\psi}(k)
\end{array}\right]^{T}\left[\begin{array}{lll}
\Psi_{11} & \Psi_{12} & \Psi_{13} \\
\Psi_{12}^{T} & \Psi_{22} & \Psi_{23} \\
\Psi_{13}^{T} & \Psi_{23}^{T} & \Psi_{33}
\end{array}\right]\left[\begin{array}{c}
x(k) \\
x_{c}(k-1) \\
d_{\psi}(k)
\end{array}\right],
$$

where

$$
\begin{aligned}
\Psi_{11}= & (G+\Delta G)^{T} P(G+\Delta G)-(1-\bar{\beta})^{2}(G+\Delta G)^{T} F^{T}(F B)^{-1} F(G+\Delta G) \\
& +(1-\bar{\beta})(2-\bar{\beta}) \Delta G^{T} F^{T}(F H)^{-1} F \Delta G-P+(1-\bar{\beta}) Q-(1-\bar{\beta})^{2} F^{T}(F H)^{-1} F, \\
\Psi_{12}= & -\bar{\beta}(2-\bar{\beta})(G+\Delta G)^{T} F^{T}(F H)^{-1} F G, \\
\Psi_{22}= & \bar{\beta}(2-\bar{\beta}) G^{T} F^{T}(F H)^{-1} F G-\delta^{2} F^{T}(F H)^{-1} F-(1-\bar{\beta}) Q,
\end{aligned}
$$

and $\Psi_{13}=(2-\bar{\beta})[\bar{\beta} G+\Delta G]^{T} F^{T}, \Psi_{23}=-\bar{\beta}(2-\bar{\beta}) G^{T} F^{T}$, and $\Psi_{33}=(2-\bar{\beta}) F \boldsymbol{H}$. Letting $d_{\psi}(k)=0$, one may claim that the system is stable if

$$
\Upsilon:=\left[\begin{array}{ll}
\Psi_{11} & \Psi_{12} \\
\Psi_{12}^{T} & \Psi_{22}
\end{array}\right]<-v I
$$

where $v>0$ is a scalar variable. Introducing $F=H^{T} P$ and exploiting Lemma 2.1, the feasibility of the inequality in $(29)$ is equivalent to that of

$$
\left[\begin{array}{cccc}
\tilde{\Psi}_{11} & \star & \star & \star \\
0 & \tilde{\Psi}_{22} & \star & \star \\
\mu_{1} H^{T} P(G+\Delta G) & -\mu_{1} H^{T} P G & -H^{T} P H & \star \\
\mu_{2} H^{T} P \Delta G & 0 & 0 & -H^{T} P H
\end{array}\right]<0,
$$

where $\mu_{1}=\sqrt{\bar{\beta}(2-\bar{\beta})}, \mu_{2}=\sqrt{(1-\bar{\beta})(2-\bar{\beta})}, \bar{Q}=(1-\bar{\beta}) Q$ and

$$
\begin{aligned}
\tilde{\Psi}_{11}= & \left(G+\Delta G+H L_{1}\right)^{T} P\left(G+\Delta G+H L_{1}\right)+L_{2}^{T}\left(H^{T} P H\right) L_{2}+(1-\bar{\beta}) L_{2}^{T} H^{T} P \\
& +(1-\bar{\beta}) P H L_{2}-P+\bar{Q}+v I, \\
\tilde{\Psi}_{22}= & -\bar{Q}+v I+L_{3}^{T}\left(H^{T} P H\right) L_{3}+\delta L_{3}^{T} H^{T} P+\delta P H L_{3},
\end{aligned}
$$


in which $L_{i}, i=1,2,3$, are auxiliary variables as in Lemma 2.1. Referring to Lemma 2.2 and by letting $P:=U\left[\begin{array}{cc}P_{11} & 0 \\ 0 & P_{22}\end{array}\right] U^{T}>0$, where $0<P_{11} \in \mathbb{R}^{m \times m}$ and $0<P_{22} \in \mathbb{R}^{(n-m) \times(n-m)}$, there exists $Z \in \mathbb{R}^{m \times m}$ so that $P H=H Z$. It follows from 31 that

$$
\begin{aligned}
\tilde{\Psi}_{11}= & {\left[P(G+\Delta G)+H Z L_{1}\right]^{T} P^{-1}\left[P(G+\Delta G)+H Z L_{1}\right]+L_{2}^{T} Z^{T} H^{T} P^{-1} H Z L_{2} } \\
& +(1-\bar{\beta}) L_{2}^{T} Z^{T} H^{T}+(1-\bar{\beta}) H Z L_{2}-P+\bar{Q}+v I . \\
\tilde{\Psi}_{22}= & -\bar{Q}+v I+L_{3}^{T} Z^{T} H^{T} P^{-1} H Z L_{3}+\delta L_{3}^{T} Z^{T} H^{T}+\delta H Z L_{3} .
\end{aligned}
$$

By employing the Schur complement and Claim 1 in [22] and introducing $Z L_{i}=X_{i}, i=1,2,3$, the inequality in 30 is implied by the LMI (17).

\subsection{Boundedness characterisation}

Theorem 3.1 in the previous subsection gives a framework to design a DSMC that stabilises the NCS in (1) with consecutive packet losses. Nevertheless, Theorem 3.1 does not characterise the augmented closed-loop system states boundedness when the external disturbance exists is not considered in Theorem 3.1 and is the subject of the following theorem.

Theorem 3.2. Given $P>0, Q>0$ and $v>0$, obtained from solving the LMI in (17), then augmented closed-loop system state $\xi(k)=\left[x^{T}(k), x_{c}^{T}(k-\right.$ 1), $\left.s_{c}^{T}(k)\right]^{T}$ is exponentially mean-square bounded as follows

$$
\exists k^{\dagger}>0, \forall k>k^{\dagger}, \forall \varepsilon>0, \text { s.t. } \mathbb{E}\left\{\|\xi(k)\|^{2}\right\} \leq \frac{\lambda_{\max }(M)}{\hat{v} \underline{\lambda}} \gamma+\varepsilon,
$$

where $\underline{\lambda}=\lambda_{\text {min }}\left(\operatorname{diag}\left(\boldsymbol{P}, Q,(F \boldsymbol{H})^{-1}\right)\right), \mathbf{M}=\operatorname{diag}\left(\boldsymbol{M}_{p}, Q\right)$ with $\boldsymbol{M}_{\boldsymbol{P}}=\boldsymbol{P}+\boldsymbol{P} \boldsymbol{H}\left(\boldsymbol{H}^{T} \boldsymbol{P} \boldsymbol{H}\right)^{-1} \boldsymbol{H}^{T} \boldsymbol{P}$, and $\gamma=\varpi^{2}\|\Pi+(2-\bar{\beta}) F H\|\left\|\mathscr{D}_{b}\right\|^{2}$, and $\hat{v}>0$ and $\Pi>0$ are the solutions of the following $L M I$ :

$$
\left[\begin{array}{cccc}
(\hat{v}-v) I+\bar{\epsilon}^{T} \mathscr{G} & \star & \star & \star \\
0 & (\hat{v}-v) I & \star & \star \\
\mu_{1}^{2} H^{T} P G & -\mu_{1}^{2} H^{T} P G & -\Pi & \star \\
0 & 0 & (2-\bar{\beta}) \mathscr{E}^{T} P H & -\bar{\epsilon} I
\end{array}\right]<0
$$

where $\bar{\epsilon}$ is a positive scalar variable. 
Proof. Defining $\tilde{x}(k)=\left[\begin{array}{ll}x^{T}(k) & x_{c}^{T}(k-1)\end{array}\right]^{T}$ and referring to Lemma 4 in [20], it can be represented that

$$
2 \tilde{x}^{T}(k)\left[\begin{array}{l}
\Psi_{13} \\
\Psi_{23}
\end{array}\right] d_{\psi}(k) \leq \tilde{x}^{T}(k)\left[\begin{array}{l}
\Psi_{13} \\
\Psi_{23}
\end{array}\right] \Pi^{-1}\left[\begin{array}{l}
\Psi_{13} \\
\Psi_{23}
\end{array}\right]^{T} \tilde{x}(k)+d_{\psi}^{T}(k) \Pi d_{\psi}(k),
$$

where $\Pi>0$ has appropriate dimension. We can follow from (28), 29) and (37) to have

$$
\Delta V(\xi(k)) \leq-\tilde{x}^{T}(k)\left\{v I-\left[\begin{array}{l}
\Psi_{13} \\
\Psi_{23}
\end{array}\right] \Pi^{-1}\left[\begin{array}{l}
\Psi_{13} \\
\Psi_{23}
\end{array}\right]^{T}\right\} \tilde{x}(k)+d_{\psi}^{T}(k)\left[\Pi+\Psi_{33}\right] d_{\psi}(k) .
$$

Choosing $\Pi>0$ such that

$$
\hat{v} I<v I-\left[\begin{array}{l}
\Psi_{13} \\
\Psi_{23}
\end{array}\right] \Pi^{-1}\left[\begin{array}{l}
\Psi_{13} \\
\Psi_{23}
\end{array}\right]^{T}
$$

where $0<\hat{v}<v$, it follows from $(38)$ that

$$
\Delta V(\xi(k)) \leq-\hat{v} \tilde{x}^{T}(k) \tilde{x}(k)+d_{\psi}^{T}(k)\left[\Pi+\Psi_{33}\right] d_{\psi}(k) .
$$

Besides, the Lyapunov candidate function in 3.1 can be rearranged as

$$
\begin{aligned}
V(\xi(k)) & =\tilde{x}^{T}(k)\left[\begin{array}{cc}
M_{P} & 0 \\
0 & Q
\end{array}\right] \tilde{x}(k) \\
& \triangleq \tilde{x}^{T}(k) M \tilde{x}(k),
\end{aligned}
$$

where $M_{P}=P+P H\left(H^{T} P H\right)^{-1} H^{T} P$, and hence one may show that

$$
\lambda_{\min }(M)\|\tilde{x}(k)\|^{2} \leq V(\xi(k)) \leq \lambda_{\max }(M)\|\tilde{x}(k)\|^{2} .
$$

Also, it is clear that

$$
\underline{\lambda}\|\xi(k)\|^{2} \leq V(\xi(k)) \leq \bar{\lambda}\|\xi(k)\|^{2},
$$

where $\underline{\lambda}=\lambda_{\min }\left(\operatorname{diag}\left(P, Q,(F H)^{-1}\right)\right)$ and $\bar{\lambda}=\lambda_{\max }\left(\operatorname{diag}\left(P, Q,(F H)^{-1}\right)\right)$. Therefore, from 40) and (41), one can show that

$$
\Delta V(\xi(k)) \leq-\frac{\hat{v}}{\lambda_{\max }(M)} V(\xi(k))+\gamma,
$$


where

$$
\gamma=\varpi^{2}\|\Pi+(2-\bar{\beta}) F H\|\left\|\mathscr{D}_{b}\right\|^{2} .
$$

Eventually, from Lemma 1 in [29] and (43) one can achieve the bound given in (35). Now for obtaining $\Pi>0$ and $\hat{v}$ utilised in (35), we need to check the feasibility of $(39)$. Given $P>0, Q>0$ and $v>0$, we then use the Schur complement and Claim 1 in [22] to illustrate that the inequality in (39) is sufficed by the LMI in 36 .

Remark 2. Note that from $(29)$, it follows that: $\forall \tilde{x}(k) \neq 0$

$$
\begin{aligned}
\mathbb{E}\left\{\left.V(\xi(k+1))\right|_{d_{\psi}(k)=0} \mid \xi(k)\right\}-V(\xi(k)) & =\tilde{x}^{T}(k) \Upsilon \tilde{x}(k) \\
& <-v \tilde{x}^{T}(k) \tilde{x}(k) .
\end{aligned}
$$

One may readily show that $\mathbb{E}\left\{\left.V(\xi(k+1))\right|_{d_{\psi}(k)=0} \mid \xi(k)\right\}>0$, and therefore, from (45) and 41, it can be claimed that $\lambda_{\max }(\boldsymbol{M})>v$. Therefore

$$
\frac{\hat{v}}{\lambda_{\max }(M)}<1
$$

This makes the proof of the above theorem in line with Lemma 1 of [29].

Remark 3. Since the control input matrix $\boldsymbol{H}$ is full column rank, if $\boldsymbol{P}>0$ the columns of $\boldsymbol{H}$ and $\boldsymbol{P} \boldsymbol{H}$ are linearly independent. As a result, if $\boldsymbol{P}>0$ and $\boldsymbol{Z}$ satisfy $\boldsymbol{P H}=\boldsymbol{H Z}$, it can easily be shown that

$$
r(Z) \geq r(H Z)=r(P H) \geq r(H)=m .
$$

This indeed implies that $Z$ is invertible. Then, letting $H=U\left[\begin{array}{l}\Sigma \\ 0\end{array}\right] V^{T}$, where $V \in \mathbb{R}^{m \times m}$ and $U \in \mathbb{R}^{n \times n}$ are orthogonal matrices, and $\Sigma:=\operatorname{diag}\left(\sigma_{1}, \cdots, \sigma_{m}\right)$, with $\sigma_{i},(i=1, \cdots, m)$ denoting nonzero singular values of $\boldsymbol{H}$, we have

$$
Z=\left(V \Sigma^{-1} P_{11}^{-1} \Sigma V^{T}\right)^{-1}
$$

The novel framework employed in the proof of Theorem 3.1 provides a substantially less-conservative sufficient condition relative to the existing conditions in the field [18]. In this paper, in contrast to [18] which exploits a conservative trivial inequality [20] to handle the negative terms in $\Delta V(\xi(k))$ 
to construct a convex problem, we employ a lossless technique (according to Lemma 2.1) which indeed imposes no unnecessary conservatism on the LMI condition derived for switching function design. Additionally, as expressed in the proof of Theorem 3.1, the feasibility region of the LMI condition used for control synthesis must not be influenced by the cross-terms between $d_{\psi}(k)$ and the system state $\left(x(k), x_{c}(k-1)\right)$. This manuscript avoids using any conservative inequality to remove the cross-terms and lets the cross-terms be in the original form. On the other hand, the LMI $(36)$ is derived for the purposes of boundedness characterisation of the system state trajectories as well as the switching function, and has no effect on the achieved controller parameters, and further, the boundary layer thickness. Indeed, the solution of the LMI (36) gives only an upper bound on $\xi(k)$. Moreover, if the LMI in (17) is feasible, the LMI in (36) will definitely be feasible and the upper bound presented in (35) exists. Finally, it is worth noting that one may solve the LMIs in (17) and (36) subject to a specific criteria in order to achieve a more accurate upper bound.

Remark 4. It should be emphasised that it is, broadly speaking, a commonly adopted method in the literature of SMC (e.g. see [2]) that with a norm bounded matched disturbance (uncertainty) in the system, a bounded nonlinear part that contains a discontinuous component can be employed to induce a sliding motion on a certain sliding manifold, whilst the linear controller maintains sliding. However, in the case of applying DSMC to discrete-time systems involving exogenous disturbances, the closed-loop system should be analysed in terms of boundedness. Further, as the VSDC is considered as a bounded component in this work, in order to make the proofs of Theorem 3.1 and 3.2 more general and in the meantime briefer, we preferred to use the general $\psi(k)$ to show any nonlinear component in the controller and prove the stability and boundedness of the augmented closed-loop system in general form. We later will specify the defined parameters associated with the system's boundedness characterisation (e.g. $\gamma$ and $\varpi)$ for the certain discontinuous controller proposed in Section 4. Fig. 1 demonstrates the idea behind Theorem 3.1 and 3.2. Indeed, if the closed-loop system in the absence of the external disturbance is stable, it will remain bounded in the presence of $d(k)$. Our contribution in Section 4 is to introduce a novel VSDC for use in DSMC applying to NCSs with packet losses so that it leads to a better performance in terms of less chattering in the trajectories of system state and switching function, as well as the reduction of the boundary layer thickness. 


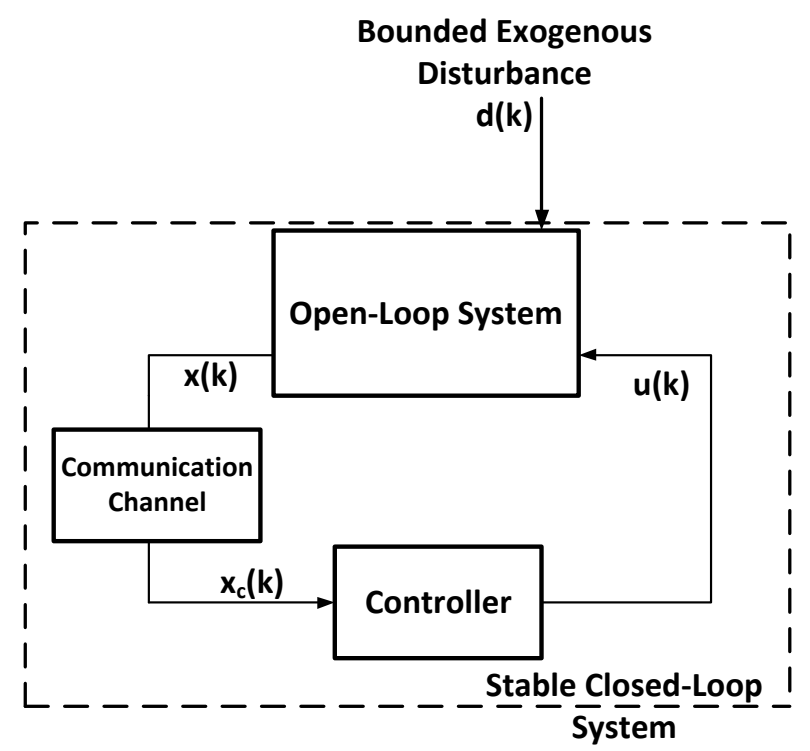

Figure 1: Schematic of the proposed control problem

\section{A Novel Variable Structure Discontinuous Controller}

As mentioned in [25], when the exogenous disturbance is smooth and bounded, $d(k-1)$ can be used as an acceptable estimate of $d(k)$. Let us temporarily assume that the system signals in (1) are not subject to packet losses, we can write

$$
d(k-1)=(F \boldsymbol{H})^{-1} F[x(k)-G x(k-1)-\Delta G(k-1) x(k-1)-H u(k-1)] .
$$

Then, $d(k-1)$ can be estimated by

$$
\hat{d}(k)=(F H)^{-1} F[x(k)-G x(k-1)-H u(k-1)] .
$$

The above equation can also be rewritten as

$$
\hat{d}(k)=(F H)^{-1} F \Delta G(k-1) x(k-1)+d(k-1) .
$$

For the balanced uncertainty $\Delta G$, one may readily show that $(F H)^{-1} F \Delta G(k-$ 1) $x(k-1)$ is also balanced and will not change the mean values of the vector $\hat{d}_{c}(k)$. Besides, while the system state is bounded, the vector $(F \boldsymbol{H})^{-1} \boldsymbol{F} \Delta \boldsymbol{G}(k-$ 
1) $x(k-1)$ remains also bounded. Hence, with the proper choice of $F$ and for the small uncertainty $\Delta \boldsymbol{G}$, it can be stated that the magnitude of $(\boldsymbol{F} \boldsymbol{H})^{-1} \boldsymbol{F} \Delta \boldsymbol{G}(k-$ 1) $x(k-1)$ would remain very small compared with that of $d(k-1)$. However, due to the packet dropout which exists in the communication network, $\hat{d}(k)$ in (46) is not applicable. Hence, the following stochastic disturbance estimator is proposed instead:

$$
\hat{d}_{c}(k)=(F H)^{-1} F\left[x_{c}(k)-G x_{c}(k-1)-H u(k-1)\right] .
$$

Then, we put the component $\psi(k)$ in $(12)$ as

$$
\psi(k)=\mathscr{D}_{a}+\frac{1}{2} \operatorname{diag}\left(\mathscr{D}_{b}\right) \operatorname{sgn}\left(\hat{d}_{c}(k)-\mathscr{D}_{a}\right),
$$

where $\operatorname{diag}\left(\mathscr{D}_{b}\right)=\operatorname{diag}\left(d_{1, b}, \cdots, d_{m, b}\right)$. In this case, $\varpi=1.5$ in (13) and $\gamma=$ $\frac{9}{4}\|\Pi+(2-\bar{\beta}) F H\|\left\|\mathscr{D}_{b}\right\|^{2}$ in 35 . Note that this bound is the worst case scenario bound and, due to slowness assumption of the exogenous disturbance in the system (1), with the perfect position estimation, this upper bound can be reduced to the one with $\varpi^{\star}=0.5$ and $\gamma^{\star}=\frac{1}{4}\|\Pi+(2-\bar{\beta}) F H\|\left\|\mathscr{D}_{b}\right\|^{2}$. Thus, the controller 12 can be chosen as

$$
u(k)=-(F H)^{-1} F G x_{c}(k)-\mathscr{D}_{a}-\frac{1}{2} \operatorname{diag}\left(\mathscr{D}_{b}\right) \operatorname{sgn}\left(\hat{d}_{c}(k)-\mathscr{D}_{a}\right),
$$

where $\hat{d}_{c}(k)$ is defined in 477$)$. Using the component (48) in the discrete-time sliding mode controller leads the state trajectories to chatter around the sliding surface with a frequency equal to the frequency of the exogenous disturbance. Moreover, for smooth and bounded exogenous disturbances, it is able to reduce the boundary layer thickness about the switching surface.

\section{Simulation Results}

In order to evaluate the proposed theory, a numerical example is given in this section. We used YALMIP [16] as well as SDPT3 [26] to solve the LMI feasibility problems. An un-interruptible power system (UPS) is considered here [29]. We aim to robustly regulates the output AC voltage of the PWM inverter at the desired setting. The capacity of UPS is 1 KVA. The sampling time $0.01 s$ at the half-load operating point is used to obtain the discrete-time 
model as:

$$
G=\left[\begin{array}{ccc}
0.9226 & -0.6330 & 0 \\
1.0 & 0 & 0 \\
0 & 1.0 & 0
\end{array}\right], H=\left[\begin{array}{l}
1 \\
0 \\
0
\end{array}\right]
$$

As seen, the open-loop system is unstable. In order to design a state-feedback controller, we assume that all the system states are available. We also consider the following modelling uncertainty parameters in the system:

$$
\begin{aligned}
\mathscr{E} & =\left[\begin{array}{lll}
0.05 & 0.15 & 0.08
\end{array}\right]^{T}, \mathscr{G}=\left[\begin{array}{lll}
-0.05 & 0.06 & 0.10
\end{array}\right], \\
\mathscr{F}(k) & =0.5 \sin (k) .
\end{aligned}
$$

The probability of the packet loss is $\bar{\beta}=0.1$. We also assume

$$
d(k)=0.1 \sin \left(\frac{k}{5}\right)
$$

Solving the LMI (17) yields the following results:

$$
\begin{aligned}
& P=\left[\begin{array}{ccc}
34.32 & 0 & 0 \\
0 & 32.33 & 6.99 \\
0 & 6.99 & 9.43
\end{array}\right], Q=\left[\begin{array}{ccc}
15.34 & -2.97 & -0.10 \\
-2.97 & 14.15 & 4.56 \\
-0.10 & 4.56 & 6.30
\end{array}\right] \\
& F=\left[\begin{array}{lll}
34.32 & 0 & 0
\end{array}\right], \quad v=0.08, \epsilon=33.49
\end{aligned}
$$

Further employing the achieved switching function matrix $F$ along with $\mathscr{D}_{a}=$ 0 and $\mathscr{D}_{b}=0.1$, we apply the control law in 49 to the system (1). Figs. 2,3 demonstrate the achieved results. Further, $x(0)=\left[\begin{array}{lll}1 & 0 & 0\end{array}\right]^{T}$. Bernoulli sequence $\beta(k)$ is indicated in Fig. 4 . Fig. 5 demonstrates the performance of the novel disturbance estimator proposed in 47 .

A Comparison: Let us apply the following controller:

$$
u(k)=-(F \boldsymbol{H})^{-1} F G x_{c}(k)-\mathscr{D}_{a}-\operatorname{diag}\left(\mathscr{D}_{b}\right) \operatorname{sgn}\left(s_{c}(k)\right),
$$

which uses a similar switching component as in [18], to the system (11). Since the LMI condition in [18] is not feasible, we construct the controller (50) exploiting the switching function matrix $F$ achieved through solving the LMI in (17). Fig. 6 demonstrates the evolution of the sliding function using the controller (50). As seen, the proposed DSMC in (49) that uses the practical switching function (48), by employing the proposed disturbance estimator 

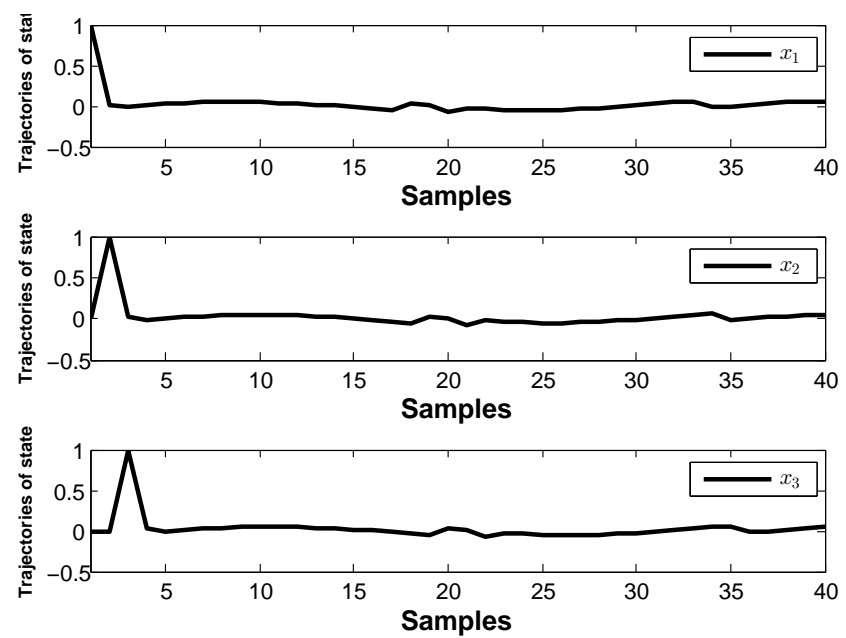

Figure 2: Evolution of the closed-loop system state trajectories using the controller 49

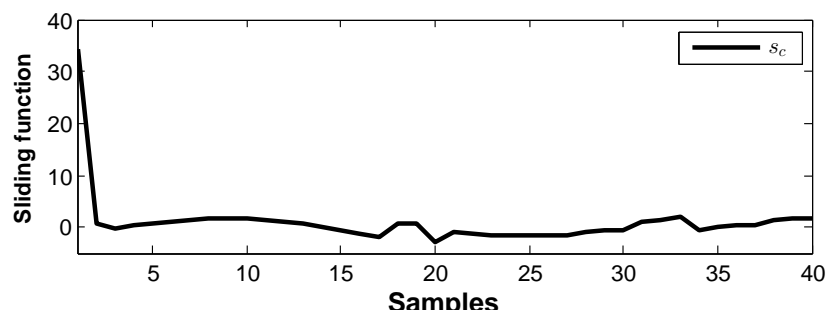

Figure 3: Evolution of the switching function using the controller 49

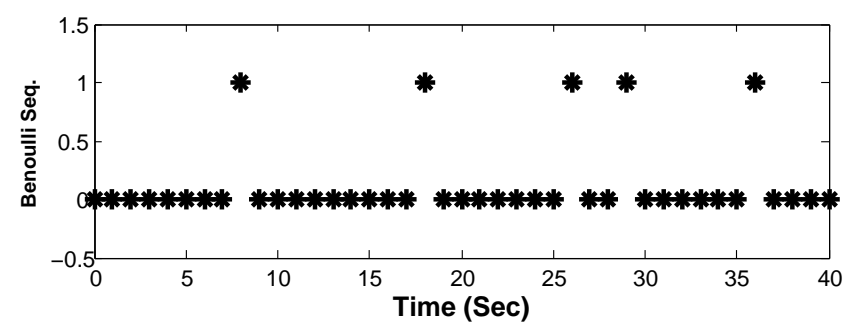

Figure 4: Bernoulli sequence $\beta(k)$ 

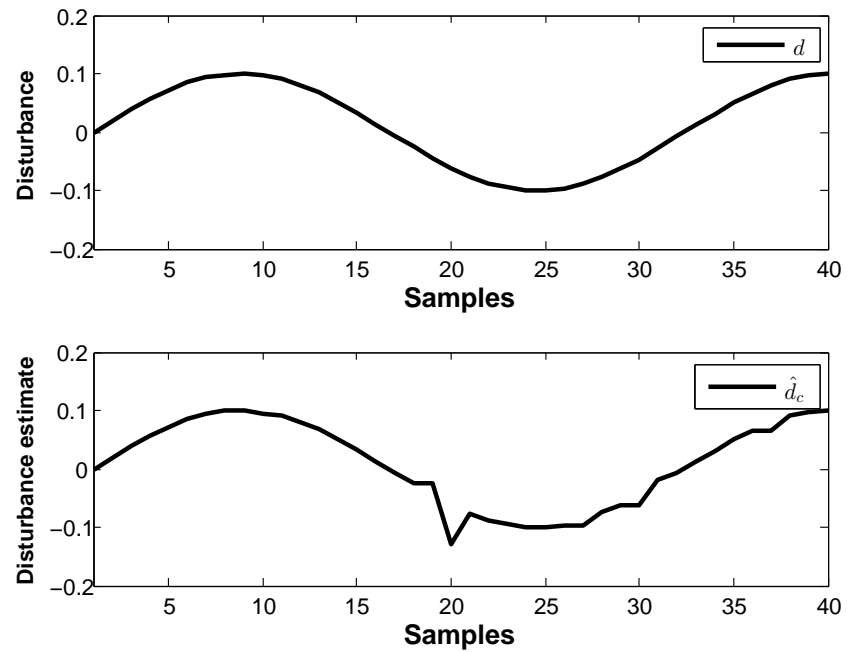

Figure 5: a) Disturbance b) Disturbance estimator output

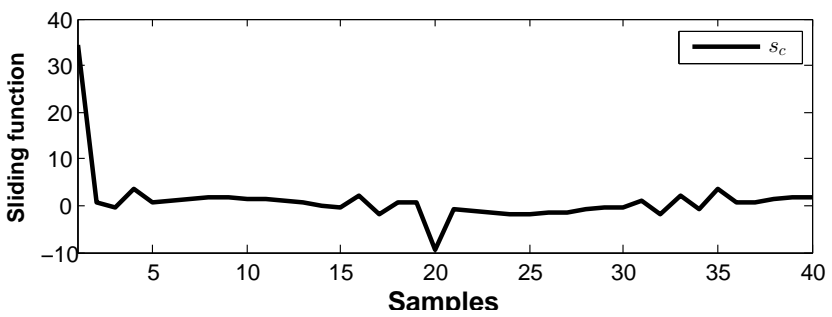

Figure 6: Evolution of the switching function using the controller (50) 
47), has effectively better performance in terms of boundary layer thickness reduction in comparison to the one in (50). Notice that in cases that there is no knowledge of the exogenous disturbances in the system, equipping the DSMC with a VSDC may not necessarily be beneficial and even can have harmful impact on the control performance [12, 24].

\section{Conclusions}

This paper has developed a robust stabilising SMC for networked systems subject to multiple data packet dropouts in the measurement communication channel. The proposed DSMC is constructed based on a less conservative LMI scheme, which is applicable to unstable systems. The mean-square stability notion has been employed for stability analysis and boundedness characterisation of the closed-loop system as well as the switching function.

\section{References}

[1] Bei Chen, Yugang Niu, and Yuanyuan Zou. Sliding mode control for stochastic markovian jumping systems subject to successive packet losses. Journal of the Franklin Institute, 351(4):2169-2184, 2014.

[2] C. Edwards. A practical method for the design of sliding mode controllers using linear matrix inequalities. Automatica, 40:1761-1769, 2004.

[3] C. Edwards and S. K. Spurgeon. Sliding Mode Control: Theory and Applications. Taylor and Francis, London, 1998.

[4] Paolo Frasca, Hideaki Ishii, Chiara Ravazzi, and Roberto Tempo. Distributed randomized algorithms for opinion formation, centrality computation and power systems estimation: A tutorial overview. European Journal of Control, 24:2-13, 2015.

[5] Ge Guo. Linear systems with medium-access constraint and markov actuator assignment. IEEE Transactions on Circuits and Systems I: Regular Papers, 57(11):2999-3010, 2010.

[6] Ge Guo. A switching system approach to sensor and actuator assignment for stabilisation via limited multi-packet transmitting channels. International Journal of Control, 84(1):78-93, 2011. 
[7] Ge Guo and Hui Jin. A switching system approach to actuator assignment with limited channels. International Journal of Robust and Nonlinear Control, 20(12):1407-1426, 2010.

[8] Ge Guo, Zibao Lu, and Qing-Long Han. Control with markov sensors/actuators assignment. IEEE Transactions on Automatic Control, 57(7):1799-1804, 2012.

[9] Ge Guo, Zibao Lu, and Peng Shi. Event-driven actuators: to zero or to hold? International Journal of Robust and Nonlinear Control, 24(17):2761-2773, 2014.

[10] G. Herrmann, S. K. Spurgeon, and C. Edwards. A robust sliding-mode output tracking control for a class of relative degree zero and nonminimum phase plants: A chemical process. International Journal of Control, 72:1194-1209, 2001.

[11] D. W. C. Ho and G. Lu. Robust stabilization for a class of discrete-time nonlinear systems via output feedback: The unified lmi approach. Int. J. Control, 76(2):105-115, 2003.

[12] S. Hui and S. H. Zak. On discrete-time variable structure sliding mode control. Syst. Control Lett., 38:283-288, 1999.

[13] I. V. Kolmanovsky and T. L. Maizenberg. Optimal control of continuoustime linear systems with a time-varying random delay. Syst. Control Lett., 44(2):119-126, 2001.

[14] N. O. Lai, C. Edwards, and S. K. Spurgeon. Discrete output feedback sliding-mode control with integral action. Int. J. Robust Nonlinear Control, 16(1):21-43, 2006.

[15] L. Li, V. A. Ugrinovskii, and R. Orsi. Decentralized robust control of uncertain Markov jump parameter systems via output feedback. Automatica, 43(11):1932-1944, 2007.

[16] J. Löfberg. YALMIP: A toolbox for modeling and optimization in MATLAB. In $C C A / I S I C / C A C S D$, September 2004.

[17] X. Luan, P. Shi, and F. Liu. Stabilization of networked control systems with random delays. IEEE Trans. Indust. Electron., 58(9):4323-4330, 2011. 
[18] Y. Niu and D. W. C. Ho. Design of sliding mode control subject to packet losses. IEEE Trans. Autom. Control, 55(11):2623-2628, 2010.

[19] Y. Niu, D. W. C Ho, and J. Lam. Robust integral sliding mode control for uncertain stochastic systems with time-varying delay. Automatica, 41(5):873-880, 2005.

[20] Yugang Niu, James Lam, Xingyu Wang, and Daniel WC Ho. Observerbased sliding mode control for nonlinear state-delayed systems. International Journal of Systems Science, 35(2):139-150, 2004.

[21] Ronald J Patton, Chandrasekhar Kambhampati, Alessandro Casavola, Ping Zhang, Steven Ding, and Dominique Sauter. A generic strategy for fault-tolerance in control systems distributed over a network. European journal of control, 13(2):280-296, 2007.

[22] I. R. Peterson. A stabilization algorithm for a class of uncertain linear systems. Syst. Control Lett., 8(4):351-357, 1987.

[23] A. Ray. Output feedback control under randomly varying distributed delays. J. Guid. Control Dyna., 17(4):701-711, 1994.

[24] S. K. Spurgeon. Hyperplane design techniques for discrete-time variable structure control systems. Int. J. Control, 55(2):445-456, 1992.

[25] W. Su, S.V. Drakunov, and Ü. Özgüner. An $\mathrm{O}\left(\mathrm{T}^{2}\right)$ boundary layer in sliding mode for sampled-data systems. IEEE Trans. Autom. Control, 45(3):482-485, 2000.

[26] K. C. Toh, M.J. Todd, R.H. Tütüncü, and R. H. Tutuncu. SDPT3 - a MATLAB software package for semidefinite programming. Optimization Methods and Software, 11:545-581, 1998.

[27] Z. Wang, D. W. C. Ho, and X. Liu. Robust filtering under randomly varying sensor delay with variance constraints. IEEE Trans. Circuits Syst. II, 51(6):320-326, 2004.

[28] Zidong Wang and Fuwen Yang. Robust filtering for uncertain linear systems with delayed states and outputs. IEEE Transactions on Circuits and Systems I: Fundamental Theory and Applications, 49(1):125-130, 2002. 
[29] F. W. Yang, Z. D. Wang, Y. S. Hung, and M. Gani. $\mathrm{H}_{\infty}$ control for networked systems with random communication delays. IEEE Trans. Autom. Control, 51(3):511-518, 2006.

[30] K. Yasuda and Y. Nakatsuji. Robust sliding mode control of uncertain systems. IEEE Workshop on Variable Structure Systems, Tokyo, Japan, 1996.

[31] Bao-Lin Zhang and Qing-Long Han. Network-based modelling and active control for offshore steel jacket platform with tmd mechanisms. Journal of Sound and Vibration, 333(25):6796-6814, 2014.

[32] Bao-Lin Zhang, Qing-Long Han, Xian-Ming Zhang, and Xinghuo Yu. Sliding mode control with mixed current and delayed states for offshore steel jacket platforms. IEEE Transactions on Control Systems Technology, 22(5):1769-1783, 2014.

[33] Bao-Lin Zhang, Li Ma, and Qing-Long Han. Sliding mode $\boldsymbol{H}_{\infty}$ control for offshore steel jacket platforms subject to nonlinear self-excited wave force and external disturbance. Nonlinear Analysis: Real World Applications, 14(1):163-178, 2013.

[34] Dan Zhang, Peng Shi, and Qing-Guo Wang. Energy-efficient distributed control of large-scale systems: A switched system approach. International Journal of Robust and Nonlinear Control, 2015.

[35] Dan Zhang, Peng Shi, Wen-An Zhang, and Li Yu. Energy-efficient distributed filtering in sensor networks: a unified switched system approach. IEEE Transactions on Cybernetics, 2016.

[36] Dan Zhang, Haiyu Song, and Li Yu. Robust Fuzzy-model-based filtering for nonlinear cyber-physical systems with multiple stochastic incomplete measurements. IEEE Transactions on System, Man, and Cybernetics: Systems, 2016.

[37] Jinhui Zhang, Yuanqing Xia, and Peng Shi. Design and stability analysis of networked predictive control systems. IEEE Transactions on Control Systems Technology, 21(4):1495-1501, 2013. 